\title{
Medication Error During the Day and Night Shift on Weekdays and Weekends: A Single Teaching Hospital Experience in Riyadh, Saudi Arabia
}

\author{
Mohammed Aljuaid (D) \\ Najla Alajman ${ }^{2}$ \\ Afraa Alsafadi ${ }^{3}$ \\ Farrah Alnajjar (D) ${ }^{4}$ \\ Mashael Alshaikh (iD) ${ }^{3}$ \\ 'Department of Health Administration, \\ College of Business Administration, King \\ Saud University, Riyadh, Saudi Arabia; \\ ${ }^{2}$ Department of Rehabilitation, Sultan Bin \\ Abdualaziz Humanitarian City, Riyadh, \\ Saudi Arabia; ${ }^{3}$ Department of Pharmacy \\ Services, King Saud University Medical \\ City, Riyadh, Saudi Arabia; ${ }^{4}$ Yanbu \\ General Hospital, Ministry of Health, \\ Riyadh, Saudi Arabia
}

Background: The association between medication error incidence and time (day shift vs night shift) have not been extensively studied in Saudi Arabia, this study aimed to answer this question: is there a relationship between medication error incidence and time of the day (day shifts vs night shifts) on weekdays and weekends?.

Objective: To identify whether medication errors and their sub-categories are significantly different between day shifts, night shifts, during weekdays and weekends.

Methods: A retrospective analysis of medication errors reported by health-care practitioners from January 2018 to December 2019 through the Electronic-Occurrence Variance Reporting System (E-OVR) of a university teaching hospital in Riyadh, Saudi Arabia. Statistical analysis was used to determine the differences between the medication errors and their subcategories and day and night shifts during weekdays (from Sunday to Thursday) and weekends (Friday and Saturday).

Results: A total of 2626 medication errors were reported over 2 years from January 2018 to December 2019. The most prevalent sub-category of medication errors was prescribing errors $(55 \%)$, while the least common sub-category of medication errors was administration errors $(0.6 \%)$. There was a statistically significant difference between medication errors and day of the week. Medication errors that happened on weekdays were greater than at weekends $(\mathrm{P}=0.01)$. During weekends, medication errors were more likely to occur at the night shift compared to the day shift $(\mathrm{P}<0.05)$.

Conclusion: Timing of medication errors incidence is an important factor to be considered for improving the medication use process and improving patient safety. Further researches are needed that focus on intervention to reduce these errors, especially during night shifts.

Keywords: medication errors, day shifts, night shifts, weekdays, weekends, patient safety

\section{Background}

Patients' safety is a crucial element in the process of providing health care. Unfortunately, the health-care providers themselves could violate patient safety accidentally. One of these misconducts is medication error. Medication errors (MEs) are a serious public health problem that threatens patient safety and imposes substantial costs (estimated at 42 US\$ billion worldwide annually, and nearly $1 \%$ of total global health expenditure). ${ }^{1}$

According to the United States National Coordinating Council for Medication Error Reporting and Prevention, MEs are defined as "any preventable event that may cause or lead to inappropriate medication use or patient harm while the
Correspondence: Mashael Alshaikh Department of Pharmacy Services, King Saud University Medical City, PO Box 2925(95), Riyadh, I |46 I, Saudi Arabia

Tel +9665044l 3294

Email malshaikh@ksu.edu.sa 
medication is in the control of the health care professional, patient, or consumer. Such events may be related to professional practice, health care products, procedures, and systems, including prescribing, order communication, product labelling, packaging, and nomenclature, compounding, dispensing, distribution, administration, education, monitoring, and use". 2

MEs consequences are ranged from mild damage to severe events that lead to hospitalization or even death. The Institute of Medicine estimated that MEs cause 1 of 131 outpatient and 1 of 854 inpatient deaths. ${ }^{3}$ Therefore, it is vital to understand the nature and causes of MEs and attempt to find potential solutions. ${ }^{4}$ MEs could increase the lengths of hospital stay for patients and increase morbidity and mortality. 5,6

This issue is a worldwide concern, and its prevalence is variably reported in many countries. In the UK, one study had estimated that 237 million MEs occur in the medication process in England annually in all settings: primary care $(38.4 \%)$, care homes $(41.7 \%)$, and secondary care $(19.9 \%)$. And those errors with the potential to cause moderate $(25.8 \%)$ or severe $(2.0 \%)$ harm. ${ }^{7}$ A recent Malaysian study had found that the prevalence of $\mathrm{ME}$ was calculated to be $30.5 \%{ }^{8}$ A systematic review had shown that MEs prevalence estimates ranged widely from $2 \%$ to $94 \%$. $^{9}$ Moreover, another systematic review studied the prevalence of computerized physician order entry systems-related medication prescription errors and found it ranged from $6.1 \%$ to $77.7 \%$. $^{10}$

The rates of MEs in Middle East countries varied from 0.18 to 56 per 100 medication orders “/” number of prescriptions and from 0.15 to 40 errors per 100 patient admissions. ${ }^{11}$ Another systematic review was conducted in the Gulf Cooperation Council countries noted that the studies report varying results, ranging from approximately $7 \%$ to $18 \%$ of prescriptions containing errors. ${ }^{12}$

Primary studies in Saudi Arabia had found that the rates of MEs range from $0.4 \%{ }^{13}$ to $1.5 \%{ }^{14}$ While a systematic review had found that the rates of MEs in Saudi Arabia are varied from $7.1 \%$ to $94 \%$ for prescribing. ${ }^{15}$ The incidence of prescribing errors was 3.6 per 100 prescriptions, 33.9 per 100 admissions and 76.5 per 1000 patient days, with the overall error rate in a tertiary paediatrics inpatient setting in Saudi Arabia was 56 per 100 medication orders. ${ }^{12}$ The highest rate of MEs was $5.5 \%$ found in intensive care units (ICUs). ${ }^{16}$ Most of MEs were for inpatient prescriptions. ${ }^{17}$ For every 100 prescriptions, there were 1.5 medication errors. ${ }^{14}$
Several factors contribute to these errors, such as system failure and the attitudes of health-care professionals. ${ }^{4}$ System failure includes a lack of the following: adequate staffing, appropriate physical and working environment, organizational communication channels, and incident reporting culture. ${ }^{18}$ Individual factors that contribute to errors include lack of general knowledge about medications, failure to adhere to policy and procedure documents, and the number of hours per shift. ${ }^{18}$ Insufficient staffing levels and increased workload were identified by healthcare practitioners as the main contributing factors for MEs. ${ }^{13}$ Also, job demands, such as social stressors (home life disruption, difficulties with colleagues), time pressures, and structural determinants (compulsory night/ weekend call duties) were significantly associated with MEs. ${ }^{19}$ Moreover, the perception amongst health-care specialists and patients is that off-hours are a riskier time to receive care. ${ }^{20}$ Other studies had shown that nurses who worked in the night shift reported a higher MEs rate due to sleep deprivation ${ }^{21}$ and work-related stress. ${ }^{19}$

A qualitative study was conducted in a 450-bed Australian teaching hospital had found that the errors were more likely to occur during tasks being carried out after hours or on long shifts. ${ }^{22}$ Another study found that more errors occurred during the evening shift (2-8 p.m.) than the night ( 8 p.m. to 8 a.m.) and morning ( 8 a.m. to 2 p.m.) shifts: $42.7 \%$ vs $28.7 \%$ and $16.7 \%$, respectively. The highest rate of death-associated errors $(4.3 \%)$ occurred in the middle of the night shift. ${ }^{23}$ A functional magnetic resonance imaging (fMRI) study revealed that task performance in non-optimal times of the day (eg, shift workers), may result in cognitive impairments leading to increased error rates and slower reaction times. ${ }^{24}$ Limited studies had investigated the association between MEs and the time of the shift (day vs night) during the week (weekday vs weekend) in Saudi Arabia. Thus, this study aims to identify whether the MEs and their sub-categories are significantly different between day and night shifts during weekdays and weekends.

\section{Method}

\section{Study Design}

This study is a retrospective analysis of medication errors reported by health-care practitioners over 2 years (Jan 2018 to December 2019) through the ElectronicOccurrence Variance Reporting System (E-OVR) of King Saud University Teaching Hospital in Riyadh, Saudi 
Arabia. The hospital is a multi-disciplinary facility with general and subspecialty medical services that provide primary, secondary, and tertiary care to its patients.

\section{Reporting System}

Medication error surveillance at King Saud Medical City was achieved through the Electronic-Occurrence Variance Reporting System (E-OVR). The reporting system is reachable through the hospital intranet, and all healthcare authorities are encouraged to report any potential or actual occurrences that might result in injury or harm in the medication use processes, which includes ordering, dispensing, and administration. The medications are passed through several steps until it reaches the patient, by as a beginning, prescribing the medication through the physician than the order passes by the system to the pharmacy where it is checked, prepared and dispensed, later the medication will be administered by nurses after comparing the medication with the physician order. This process is the routine that is done through the weekdays and weekends.

Any medication error discovered through this process will be reported to the E-OVR system that will be immediately sent to the investigator. This person will start investigating using different tools; starting by checking the reported medication error with the patient electronic file to ensure that all data are correct and to exclude any reports that are not considered medication error as adverse drug reactions, allergy, overdose, drug interaction.

Then, the investigator makes an interview with the involved staff to understand the causes of the error, finally the investigator makes a tour to the location where the error had happened to observe the area. These tools are used by the investigator to find and extract the causes of these errors that will be discussed with the supervisor or in charge of the intended area, to put an action to prevent further errors and avoid patient harm.

The electronic form of medication error reporting includes the following information: date and time of incidence, location of the error (unit/ward of the hospital), and categories (medication errors, adverse drug reaction, and others). Sub-categories for each main category were also recorded: medication errors include prescribing, dispensing, and administration. Prescribing errors including prescription to a patient with documented allergy, contraindicated drug, wrong dose/strength, wrong medication, and duplication therapy. Dispensing errors containing; wrong medication and wrong label.
For the administration errors, it could be wrong dose, wrong time, and wrong documentation. Other subcategories were availability of the medications, delayed medication, expired medication, no dispensing after order the medication, and storage condition.

\section{Data Collection}

Medication error forms were collected from the Reporting System (E-OVR) of King Saud University Hospital. All the incidents that were reported by health-care practitioners from January 1st, 2018 to December 31st, 2019 included in this study.

Each dataset (2018 and 2019) was entered into a separate Excel sheet. The following variables were extracted for each incident: time, categories of MEs (adverse drug reactions excluded from the beginning, since the main focus of this study on MEs), sub-categories of MEs (prescribing, dispensing, and administration), other medication errors (availability, delayed medication, expired medication, no dispensing after order, and storage condition). The eight variables were available for each dataset, and then both datasets were merged based on the variable names, which were similar in both sheets.

\section{Data Analysis}

Statistical analysis was performed using the Statistical Package for the Social Sciences (SPSS version 25). Data were entered into the SPSS sheet, cleaned, and re-coded. Descriptive statistics were used to summarize the data. Descriptive statistics with frequencies and per cent were used for category and sub-category of MEs. The $\mathrm{Z}$ test of independence was used to determine whether there are significant differences between the sub-category of MEs and the shift work that was broken down into two time periods: a nighttime shift from $7 \mathrm{pm}$ to $7 \mathrm{am}$, daytime shift 7 am to $7 \mathrm{pm}$, during weekdays (Sunday to Thursday) and weekends (Friday and Saturday). Comparisons of MEs between weekdays and weekends as well as between the day and night shifts were assessed using an independent sample $t$-test and a p-value less than 0.05 was considered statistically significant. All data relevant to the study are included in the article and could be further explained on request from the corresponding author.

\section{Results}

A total of 2626 MEs were reported for the 2 years from January 2018 to December 2019. The most prevalent sub- 
category of MEs was prescribing errors (55\%), followed by a lack of availability (14.1\%) and delayed medications $(10.1 \%)$. The least common sub-categories of MEs were not giving medications after ordering $(0.8 \%)$ and administration errors $(0.6 \%)$ (Table 1$)$.

Furthermore, Table 1 shows the number and percentages for each sub-category of MEs during the day and night shifts on weekdays and weekends. There were statistically significant differences between sub-categories of MEs in day and night shifts during weekdays and weekends $(\mathrm{P}<0.05)$, except the "No dispensing after order" and "Administration" items as well as "Expired medication" during weekends. The highest percentage of the prescribing errors occurred during night shifts on weekdays and weekends (76.7\% vs $79.3 \%$ ) respectively, followed by a lack of availability ( $6.8 \%$ vs $6.7 \%)$, then delayed medication (5.1\%) on weekdays and the storage condition $(3.9 \%)$ on weekends. No dispensing after order contributed the fewest errors $(0.8 \%$ vs $1.1 \%)$.

As shown in Table 2, there was a statistically significant difference between MEs and the day of the week. MEs that occurred on weekdays were significantly higher than at weekends $(\mathrm{P}=0.01)$. However, there was no significant difference between day and nights shifts and also between the two shifts during weekdays. Further, it was observed that the occurrences of MEs in night shifts were significantly higher than those in day shifts during weekends $(\mathrm{P}<0.05)$.

Figure 1 shows the average monthly number of MEs per day during weekdays and weekends. The monthly MEs that occurred on weekdays were higher than the weekends, except for November and December 2018, the number of errors that occurred on weekends were greater ( 8.38 vs 8.05 and 8.13 vs 7.36 ) respectively. The highest number of MEs occurred during those two months of 2018 for both weekdays and weekends.

\section{Discussion}

The aim of the present study is to identify whether the incidents of MEs and its sub-categories are significantly different between day and night shift during weekdays and weekends in a teaching hospital in Saudi Arabia.

The current study showed that there is a statistically significant relationship between MEs and the day of the week (weekday and weekend). Overall, the MEs were higher during the weekdays than at weekend. However, during the weekends, MEs at the night shifts were significantly higher than the day shift. The possible explanations for this are the limited number of staff during night shifts and the weekend, thus that may limit the volunteering reporting ability so lesser incidents would be reported at night shifts and weekends. On the other hand, in the morning and weekdays shifts, the

Table I Sub-Categories of Medication Errors and Time

\begin{tabular}{|c|c|c|c|c|c|c|c|c|c|}
\hline \multirow{2}{*}{$\begin{array}{l}\text { Sub-Categories of } \\
\text { Medication Error }\end{array}$} & \multirow{2}{*}{$\begin{array}{l}\text { Total } \\
\text { N (\%) }\end{array}$} & \multicolumn{3}{|c|}{ Weekdays } & \multirow[t]{2}{*}{$\mathbf{P}$} & \multicolumn{3}{|c|}{ Weekends } & \multirow[t]{2}{*}{$\mathbf{P}$} \\
\hline & & Total & $\begin{array}{l}\text { Day Shift } \\
\text { N (\%) }\end{array}$ & $\begin{array}{l}\text { Night } \\
\text { Shift } \\
\text { N (\%) }\end{array}$ & & Total & $\begin{array}{l}\text { Day Shift } \\
\text { N (\%) }\end{array}$ & $\begin{array}{l}\text { Night } \\
\text { Shift } \\
\text { N (\%) }\end{array}$ & \\
\hline Dispensing & $192(7.3)$ & 168 & 125 (II.9) & $43(3.8)$ & $<0.001$ & 24 & $16(10.1)$ & $8(2.8)$ & 0.001 \\
\hline Prescribing & I445 (55) & 1162 & $296(28.1)$ & 866 (76.7) & $<0.001$ & 283 & $57(35.8)$ & $226(79.3)$ & $<0.001$ \\
\hline Availability & $37 \mid(14.1)$ & 317 & $240(22.8)$ & $77(6.8)$ & $<0.001$ & 54 & $35(22)$ & $19(6.7)$ & $<0.001$ \\
\hline Delayed medication & $264(10.1)$ & 235 & $177(16.8)$ & $58(5.1)$ & $<0.001$ & 29 & $19(11.9)$ & $10(3.5)$ & $<0.001$ \\
\hline Expired medication & $79(3)$ & 61 & $42(4.0)$ & $19(1.7)$ & $<0.001$ & 18 & $10(6.3)$ & $8(2.8)$ & 0.06 \\
\hline No dispensing after order & $20(0.8)$ & 16 & $7(0.7)$ & $9(0.8)$ & 0.359 & 4 & I (0.6) & $3(1.1)$ & 0.294 \\
\hline Storage condition & $238(9)$ & 206 & $160(15.2)$ & $46(4.1)$ & $<0.001$ & 32 & $21(13.2)$ & II (3.9) & $<0.001$ \\
\hline Administration & $17(0.6)$ & 17 & $6(0.6)$ & II (I.0) & 0.142 & - & - & - & - \\
\hline Total & $2626(100)$ & 2182 & $\begin{array}{l}1053 \\
(100)\end{array}$ & $1129(100)$ & - & 444 & $159(100)$ & $285(100)$ & - \\
\hline
\end{tabular}


Table 2 Comparisons of Medication Errors Between Day and Night Shifts During Weekdays and Weekends

\begin{tabular}{|l|l|l|l|}
\hline Time & Mean \pm SD & t Statistic & $\begin{array}{l}\text { P value } \\
\text { (Two } \\
\text { Tailed) }\end{array}$ \\
\hline $\begin{array}{c}\text { Day of week } \\
\text { Weekdays } \\
\text { Weekends }\end{array}$ & $\begin{array}{l}4.8 \pm 4.9 \\
3.2 \pm 4.9\end{array}$ & 3.2 & 0.001 \\
\hline $\begin{array}{c}\text { Shifts } \\
\text { Day } \\
\text { Night }\end{array}$ & $\begin{array}{l}1.67 \pm 2.3 \\
1.94 \pm 4.3\end{array}$ & -1.543 & 0.123 \\
\hline $\begin{array}{c}\text { Shift of weekdays } \\
\text { Day } \\
\text { Night }\end{array}$ & $2.3 \pm 2.5$ & -0.6 & 0.493 \\
\hline $\begin{array}{c}\text { Shift of weekends } \\
\text { Day } \\
\text { Night }\end{array}$ & $\begin{array}{l}1.1 \pm 1.4 \pm 4.6 \\
2.1 \pm 4.9\end{array}$ & -2.1 & 0.038 \\
\hline
\end{tabular}

hospital working capacity would be at its maximum which may reflect positively on the reporting system. ${ }^{25}$

Our findings are in line with the results of Miller et al, who compared reported MEs rates over a 1-year period in a children's hospital and had found that the error rate on night shift was higher than the error rate on day shifts. ${ }^{20}$ Moreover, the findings of this study are consistent with those in an earlier report by Niu et al, which showed that the night shift workers had significantly higher error rates. ${ }^{26}$ In addition, in KSA, Salam et al who examined the relationship between the overall levels of stress and
MEs rate, had shown that MEs are significantly higher amongst health practitioners who work in the night shift "all the time" $(\mathrm{P}=0.004)$ compared with those who work in the day shift. ${ }^{19}$

This is similar to the findings of a cross-sectional survey conducted with residents enrolled in paediatrics residency programs in Saudi Arabia, where the authors found that 24-hrs on-call system negatively impacts residents' performance during night duty due to fatigue, residents' overall well-being, and patient care. ${ }^{27}$

However, a study conducted in a tertiary hospital in Jeddah describing 624 medication error extracted from the hospital safety reporting system, had found that ME incidents were more likely to be reported in the morning shift versus evening and night shift (77.4 vs 22.6). ${ }^{25}$ A possible explanation for these results may be due to stress, fatigue and sleep deprivation. Since multiple studies indicated a significant relationship between these factors and MEs among healthcare practitioners, Arimura et al conducted a study in Japan to examine the relationship between shift work, mental health, and self-reported medical errors among hospital nurses, and they concluded that shift work and poor mental health were significant factors contributing to medical errors. ${ }^{28}$ Nevertheless, further investigations are needed since fatigue and sleep deprivation contributed to more errors in the night shift among nurses, ${ }^{21,29,30}$ which is in line with the results of our study in terms of increased ME rates during night shifts.

As shown in Figure 1, the current study showed that in November and December 2018 the number of MEs that occurred on weekends were greater than the other months.

\section{Medication errors during weekdays and weekends}

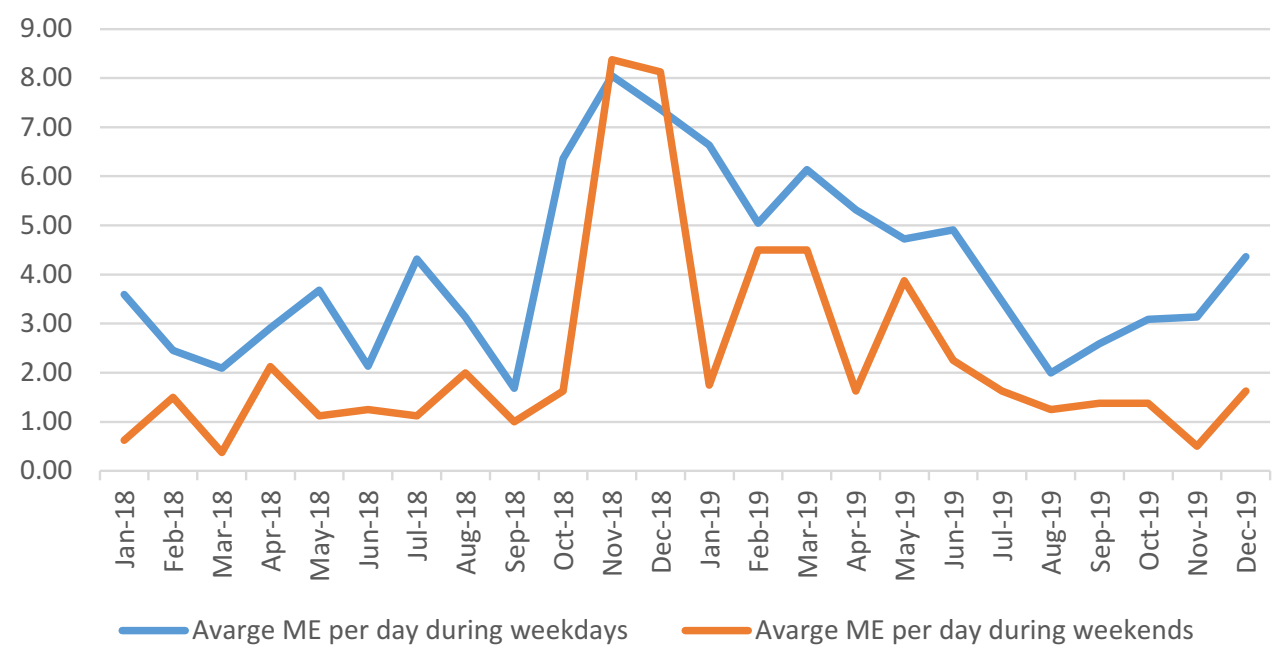

Figure I Monthly number of medication errors during weekdays and weekends (2018-2019). 
These results are consistent with Kuitunen et al study who found that MEs are more likely to occur during holiday seasons since the number of MEs was greatest during the summer months and in December. ${ }^{31}$ Kuitunen et al contributed this high number in the holidays seasons to the low number of permanent staff and a high number of temporary replacements. ${ }^{31}$ Although the Christmas season is not popular in Saudi Arabia since it is not part of the culture, a high percentage of the hospital staffs are nonSaudi and these results are applicable as well.

Among the sub-categories of MEs, prescribing errors were occurred at a higher rate during night shifts on weekdays and weekends (76.7 vs 79.3) respectively with $(\mathrm{P}<$ 0.001).

Administration errors accounted for only $0.6 \%$ similar to the earlier report by Ali et al which showed that the fewest errors occurred at the dispensing and administration stages. ${ }^{14}$ Earlier study by Leape et al showed that administration errors occur frequently as much as $27 .^{32}$ This is opposite to our finding which shows administration errors occurs by 0.6 . The under-reporting issue in our local hospitals might explain our findings. In 2013, Al Shaikh et al had conducted a study in a hospital in Riyadh, Saudi Arabia - which is the same setting as this study - and the authors concluded that MEs were underreported in this hospital. ${ }^{13}$ Moreover, Eltaybani et al had found a statistically significant relationship $(p<0.001)$ between error disclosure and the error impact on the involved patients; the less the significance of induced patients' harm, the less the frequency of error disclosure. ${ }^{23}$ They concluded that nurses are more likely to report their errors when they feel safe and when the reporting system is not burdensome. $^{23}$

Under-reporting of MEs have been documented in all countries, particularly in developing countries. ${ }^{33}$ Identifying reasons for under-reporting is important to prevent these incidences. ${ }^{33}$

Tobaiqy and Stewart identified three key barriers to reporting errors: lack of awareness of the reporting policy; workload and time constraints associated with reporting; and unavailability of the reporting form. ${ }^{34}$ The main barriers to the reporting of errors by nurses were related to the administrative response, fear of reporting, and disagreements regarding the definitions of errors. ${ }^{35}$ Furthermore, some physicians feared punitive actions if they reported errors; a culture of blame remains predominant among most health-care providers. ${ }^{33}$
Ross et al mentioned that the lack of personal knowledge, experience, workload, and time pressures were the factors related to prescribing errors by junior doctors. ${ }^{36}$ Also, the cognitive burden was explained in two ways: physical, associated with fatigue, distraction and interruption, and poor information transfer; and psychological, related to inexperience, changing workload, and insufficient decision-support information. ${ }^{37}$

Niu et al suggested some recommendations to minimize ME rate, that more than 2 days off when shifting from the night shift to other shifts essential to provide enough time to adjust daily rhythm. ${ }^{26}$ Zion and Shochat found that a scheduled nap has a positive impact on the consequences of night shift work in female nurses. ${ }^{38}$

We encountered several limitations when conducting this study. First, the study was conducted in one centre, which might affect the generalizability of the results. The second limitation is related to the voluntary reporting by health-care providers that depend on their awareness, which leads to underreporting. The setting of this study is King Saud University Teaching Hospital in Riyadh, Saudi Arabia, the hospital employed different nurses' nationalities with different cultures, and that will affect the reporting response of administration errors. Some of the health-care providers fear reporting and punitive actions or they are embarrassed by their mistakes and may try to conceal them or defend themselves by shifting the blame to someone or something else. Furthermore, this voluntary reporting system might need additional time for reporting MEs.

The MEs reporting process can be improved by creating a just working culture, support from administration, and significant feedback on reported errors. ${ }^{13}$ The implementation of a "culture of safety" and "just culture" might help the staff to understand the importance of patient safety and further enhanced reporting of medication errors. ${ }^{16}$ The health-care organization should adopt a program that enhances and promotes error reporting, based on transparency, confidentiality, and no-blame culture. ${ }^{33}$

Electronic prescribing systems help to reduce medication errors, although they do not prevent all errors. Segal and colleagues had programmed a medication-dosing app using a mobile health tool (CommCare). ${ }^{39}$ They assessed the app's dosing accuracy and efficiency, and then they measured the app's usability and effect on patientcenteredness. The app was shown to be safe and efficient. Making this app available to junior physicians might significantly improve patient safety by enhancing dosing accuracy. ${ }^{39}$ 
Night shift work could contribute to fatigue and sleep problems that might then affect the performance of healthcare professionals. Therefore, the examination of the relationship between medication errors and time of occurrence is an essential step in maintaining the quality of service provided to the patients, thus maintaining their safety.

There is a need for further investigation on how to improve the prescribing medication information to decrease these errors, such as introducing a mobile app to ensure the correct dose. Also, studying the influence of the level of experience of the prescriber on the error. Further studies are needed to examine the association between the area of specialities and high MEs rates, especially in ICUs and paediatrics.

\section{Conclusion}

The most prevalent sub-category of MEs was prescribing errors $(55 \%)$ which occurred mostly during night shifts on weekdays and weekends. Moreover, the monthly MEs that occurred on weekdays were higher than at the weekends. These findings show the timing as an important factor to improve the medication use process and to improve patient safety by using all available tools. Also, to assess the need for additional strategies that aiming error prevention. Further research is needed to investigate which specialities contribute most to MEs and the relationship of that with time (night shifts, day shifts, weekdays and weekends), and how to improve the prescribing medication process, as well as studies that focus on intervention or potential solution to reduce these errors during night shifts.

\section{Ethical Consideration}

Ethical approval was obtained from King Saud University with the number KSU-HE-20-209. The data are anonymous, and the requirement for informed consent was therefore waived. This study was conducted in accordance with the Declaration of Helsinki.

\section{Acknowledgment}

The authors are grateful to the Research Centre, College of Business Administration and the Deanship of Scientific Research at the King Saud University for supporting this study. We are also grateful to King Saud University Medical City and all employees of the associations who supported data collection.

\section{Author Contributions}

All authors made substantial contributions to conception and design, acquisition of data, or analysis and interpretation of data; took part in drafting the article or revising it critically for important intellectual content; agreed to submit to the current journal; gave final approval of the version to be published; and agree to be accountable for all aspects of the work.

\section{Funding}

The authors extend their appreciation to the Deputyship for Research \& Innovation, "Ministry of Education" in Saudi Arabia for funding this research work through the project number IFKSURG-274.

\section{Disclosure}

The authors report no conflicts of interest in this work.

\section{References}

1. Aitken M, Gorokhovich L. Advancing the responsible use of medicines: applying levers for change. SSRN 2222541; 2012.

2. Prevention. NCCfMERa, error Wiam. About medication error 2015. Available from: https://www.nccmerp.org/about-medication-errors. Accessed June 7, 2021.

3. Wittich CM, Burkle CM, Lanier WL. Medication errors: an overview for clinicians. Mayo Clinic Proceedings. Elsevier; 2014.

4. Abubakar AR, Chedi BA, Simbak NB, Haque M. Medication error: the role of health care professionals, sources of error and prevention strategies. J Chem Pharm Res. 2014;6(10):646-651.

5. Hussain E, Kao E. Medication safety and transfusion errors in the ICU and beyond. Crit Care Clin. 2005;21(1):91-110, ix. doi:10.1016/ j.ccc.2004.08.003

6. Barker KN, Flynn EA, Pepper GA, Bates DW, Mikeal RL. Medication errors observed in 36 health care facilities. Arch Intern Med. 2002;162(16):1897-1903. doi:10.1001/archinte.162.16.1897

7. Elliott RA, Camacho E, Jankovic D, Sculpher MJ, Faria R. Economic analysis of the prevalence and clinical and economic burden of medication error in England. BMJ Qual Saf. 2021;30(2):96-105. doi:10.1136/bmjqs-2019-010206

8. Shitu Z, Aung MM, Kamauzaman TH. Prevalence and characteristics of medication errors at an emergency department of a teaching hospital in Malaysia. BMC Health Serv Res. 2020;20(1):1-7. doi:10.1186/s12913-020-4921-4

9. Assiri GA, Shebl NA, Mahmoud MA, et al. What is the epidemiology of medication errors, error-related adverse events and risk factors for errors in adults managed in community care contexts? A systematic review of the international literature. BMJ Open. 2018;8(5):e019101. doi:10.1136/bmjopen-2017-019101

10. Korb-Savoldelli V, Boussadi A, Durieux P, Sabatier B. Prevalence of computerized physician order entry systems-related medication prescription errors: a systematic review. Int $J$ Med Inform. 2018;111:112-122. doi:10.1016/j.ijmedinf.2017.12.022

11. Thomas B, Paudyal V, MacLure K, et al. Medication errors in hospitals in the Middle East: a systematic review of prevalence, nature, severity and contributory factors. Eur J Clin Pharmacol. 2019;75(9):1269-1282. doi:10.1007/s00228-019-02689-y

12. Alsaidan J, Portlock J, Aljadhey HS, Shebl NA, Franklin BD Systematic review of the safety of medication use in inpatient, outpatient and primary care settings in the Gulf Cooperation Council countries. Saudi Pharm J. 2018;26(7):977-1011. doi:10.1016/j. jsps.2018.05.008 
13. Alshaikh M, Mayet A, Aljadhey H. Medication error reporting in a university teaching hospital in Saudi Arabia. J Patient Saf. 2013;9 (3):145-149. doi:10.1097/PTS.0b013e3182845044

14. Ali S, Aboheimed NI, Al-Zaagi IA, Al-Dossari DS. Analysis of medication errors at a large tertiary care hospital in Saudi Arabia: a retrospective analysis. Int J Clin Pharm. 2017;39(5):1004-1007. doi:10.1007/s11096-017-0514-7

15. Alhomoud FK, Alnemari W, Alfahmi H, Alhomoud F, Cheema E. Incidence and prevalence of prescribing errors in Saudi Arabia: a systematic study. Int $J$ Pharm Pharm Sci. 2016;8(12):194-198. doi:10.22159/ijpps.2016v8i12.14973

16. Al Harbi SA, Al-Qhtani NM, Bustami R, et al. A comparative study of voluntarily reported medication errors among adult patients in intensive care (IC) and non-IC settings in Riyadh, Saudi Arabia. Trop J Pharm Res. 2016;15(12):2713-2718. doi:10.4314/tjpr. v15i12.25

17. Alshahrani SM, Alakhali KM, Al-Worafi YM. Medication errors in a health care facility in southern Saudi Arabia. Trop J Pharm Res. 2019;18(5):1119-1122. doi:10.4314/tjpr.v18i5.29

18. McBride-Henry K, Foureur M. Medication administration errors: understanding the issues. Aust $J$ Adv Nurs. 2006;23(3):33.

19. Salam A, Segal DM, Abu-Helalah MA, et al. The impact of work-related stress on medication errors in Eastern Region Saudi Arabia. Int J Qual Health Care. 2019;31(1):30-35. doi:10.1093/ intqhe/mzy097

20. Miller AD, Piro CC, Rudisill CN, Bookstaver PB, Bair JD, Bennett CL. Nighttime and weekend medication error rates in an inpatient pediatric population. Ann Pharmacother. 2010;44 (11):1739-1746. doi:10.1345/aph.1P252

21. Ramadan MZ, Al-Saleh KS. The association of sleep deprivation on the occurrence of errors by nurses who work the night shift. Curr Health Sci J. 2014;40(2):97. doi:10.12865/CHSJ.40.02.03

22. Nichols P, Copeland TS, Craib IA, Hopkins P, Bruce DG. Learning from error: identifying contributory causes of medication errors in an Australian hospital. Med J Aust. 2008;188(5):276-279. doi:10.5694/ j.1326-5377.2008.tb01619.x

23. Eltaybani S, Mohamed N, Abdelwareth M. Nature of nursing errors and their contributing factors in intensive care units. Nurs Crit Care. 2019;24(1):47-54. doi:10.1111/nicc.12350

24. Reske M, Rosenberg J, Plapp S, Kellermann T, Shah NJ. fMRI identifies chronotype-specific brain activation associated with attention to motion-Why we need to know when subjects go to bed. Neuroimage. 2015;111:602-610. doi:10.1016/j. neuroimage.2015.02.013

25. Aseeri M, Banasser G, Baduhduh O, Baksh S, Ghalibi N. Evaluation of medication error incident reports at a tertiary care hospital. Pharmacy. 2020;8(2):69. doi:10.3390/pharmacy8020069

26. Niu S-F, Chu H, Chen C-H, et al. A comparison of the effects of fixed-and rotating-shift schedules on nursing staff attention levels: a randomized trial. Biol Res Nurs. 2013;15(4):443-450. doi:10.1177/ 1099800412445907

Risk Management and Healthcare Policy

\section{Publish your work in this journal}

Risk Management and Healthcare Policy is an international, peerreviewed, open access journal focusing on all aspects of public health, policy, and preventative measures to promote good health and improve morbidity and mortality in the population. The journal welcomes submitted papers covering original research, basic science, clinical \& epidemiological studies, reviews and evaluations,
27. Alsohime FM. Pediatric residents' perceptions of the impact of the 24-hour on-call system on their well-being and education and patient safety. Saudi Med J. 2019;40(10):1040-1044. doi:10.15537/ smj.2019.10.24548

28. Arimura M, Imai M, Okawa M, Fujimura T, Yamada N. Sleep, mental health status, and medical errors among hospital nurses in Japan. Ind Health. 2010;48(6):811-817. doi:10.2486/indhealth. MS1093

29. Johnson AL, Jung L, Brown KC, Weaver MT, Richards KC. Sleep deprivation and error in nurses who work the night shift. J Nurs Adm. 2014;44(1):17-22. doi:10.1097/NNA.0000000000000016

30. Surani S, Hesselbacher S, Guntupalli B, Surani S, Subramanian S. Sleep quality and vigilance differ among inpatient nurses based on the unit setting and shift worked. J Patient Saf. 2015;11(4):215-220. doi:10.1097/PTS.0000000000000089

31. Kuitunen T, Kuisma P, Hoppu K. Medication errors made by health care professionals. Analysis of the Finnish Poison Information Centre data between 2000 and 2007. Eur J Clin Pharmacol. 2008;64 (8):769-774. doi:10.1007/s00228-008-0496-4

32. Leape LL, Bates DW, Cullen DJ, et al. Systems analysis of adverse drug events. JAMA. 1995;274(1):35-43. doi:10.1001/ jama.1995.03530010049034

33. Alsafi E, Baharoon S, Ahmed A, Al Jahdali H, Al Zahrani S, Al Sayyari A. Physicians' knowledge and practice towards medical error reporting: a cross-sectional hospital-based study in Saudi Arabia. East Mediterr Health J. 2015;21(9):655-664. doi:10.26719/ 2015.21.9.655

34. Tobaiqy M, Stewart D. Exploring health professionals' experiences of medication errors in Saudi Arabia. Int J Clin Pharm. 2013;35 (4):542-545. doi:10.1007/s11096-013-9781-0

35. Hammoudi BM, Ismaile S, Abu Yahya O. Factors associated with medication administration errors and why nurses fail to report them. Scand J Caring Sci. 2018;32(3):1038-1046. doi:10.1111/scs.12546

36. Ross S, Ryan C, Duncan EM, et al. Perceived causes of prescribing errors by junior doctors in hospital inpatients: a study from the PROTECT programme. BMJ Qual Saf. 2013;22(2):97-102. doi:10.1136/bmjqs-2012-001175

37. Sutherland A, Ashcroft DM, Phipps DL. Exploring the human factors of prescribing errors in paediatric intensive care units. Arch Dis Child. 2019;104(6):588-595. doi:10.1136/archdischild-2018-315981

38. Zion N, Shochat T. Let them sleep: the effects of a scheduled nap during the night shift on sleepiness and cognition in hospital nurses. $J$ Adv Nurs. 2019;75(11):2603-2615. doi:10.1111/jan.14031

39. Segal JB, Arevalo JB, Franke MF, Palazuelos D. Reducing dosing errors and increasing clinical efficiency in Guatemala: first report of a novel mHealth medication dosing app in a developing country. $B M J$ Innov. 2015;1(3):111-116. doi:10.1136/bmjinnov-2015-000051 